\title{
Ultra-high speed X-ray imaging of dynamic fracturing in cementitious materials under impact
}

\author{
Bratislav Lukić1,*, Maria Blasone ${ }^{2}$, Yannick Duplan ${ }^{2}$, Pascal Forquin ${ }^{2}$, Emilio Escauriza ${ }^{3,1}$, \\ Michael Rutherford ${ }^{3}$, David Chapman ${ }^{3}$, Daniel Eakins ${ }^{3}$, Margie Olbinado ${ }^{4}$, and Alexander \\ Rack $^{1}$ \\ ${ }^{1}$ ESRF - The European Synchrotron, CS 40220, F-38043 Grenoble Cedex 9, France \\ ${ }^{2}$ Univ. Grenoble Alpes, CNRS, Grenoble INP, 3SR, 38000 Grenoble, France \\ ${ }^{3}$ Department of Engineering Science, University of Oxford, Parks Road, Oxford OX1 3PJ, UK \\ ${ }^{4}$ Swiss Light Source, Paul Scherrer Institut, X-ray Tomography Group, Villigen, Switzerland
}

\begin{abstract}
In this work the dynamic fracturing of an ultra-high strength cementitious material is probed with in-situ ultra-high speed X-ray phase-contrast diagnostics to investigate the phenomenology of dynamic fracture. Gas gun experiments were conducted on two characteristic samples with two different impact speeds, namely 80 and $190 \mathrm{~m} / \mathrm{s}$ using the edge-on impact test configuration. The samples were placed within the intense $\mathrm{X}$-ray beam providing an observation field of $12.8 \mathrm{~mm}$ in width and $8 \mathrm{~mm}$ in height. Thanks to equispaced 16 bunches of short X-ray pulses, the samples were imaged through an indirect detector arrangement using the Shimadzu HPV-X2 camera lens-coupled to a fast scintillator capturing through-thickness measurements with an interframe time of $1.06 \mu \mathrm{s}$. The comparison of fragmentation patterns between two samples revealed an important insight into velocity dependant spall formation as well as the effects of crack closure and bridging.
\end{abstract}

\section{Introduction}

Under dynamic loading conditions, brittle solids fail by exhibiting a multiple fracturing phenomenon with a transition from a probabilistic failure, such as in the case of a single quasi-static crack, towards the deterministic failure where many cracks initiate and propagate at the same time [1, 2]. On a microscale, the cracks initiate from inherent flaws and grow to eventually coalesce and form macrocracks. During this process, dynamic cracks can propagate as fast as the material shear wave speed [3], leading to crack interactions such as obscuration, coalescence, bridging and bifurcation [4]. Several numerical strategies have been developed in an attempt to simulate the complex phenomena of dynamic crack formation and bifurcation, either through continuum or discrete framework (e.g [5-7]). However, the coalescence of dynamic fractures remains a challenging task from a numerical standpoint. Furthermore, the way in which microstructural material heterogeneities influence the path of dynamic cracks still remains an open question [8]. In order to further advance the developments of predictive numerical strategies, experimental works with rich diagnostics are paramount. Traditionally, dynamic fragmentation experiments have been diagnosed

\footnotetext{
*e-mail: lukic@esrf.fr
} 
with time-resolved surface measurements (full-field or pointwise), followed by post-mortem analysis techniques, usually through microstructural inspection of recuperated fragments (e.g. [9]). Although insightful, these approaches leave a gap regarding complex interplay of sub-surface processes, such as inception and growth of cracks and pores that are strongly influenced by the bulk material microstructure.

With the development of high-energy synchrotron photon sources, combining high brilliance, short bunch pulse duration and high-energy, X-rays can be used to probe matter during transient dynamics by capturing through volume information with high temporal and spatial resolution [10]. In this work, multiple dynamic fracturing phenomena under impact have been probed by ultra-high speed (UHS) X-ray phase contrast imaging (XPCI) using synchrotron radiation at the ID19 beamline of the European Synchrotron Radiation Facility [11]. Gas gun experiments have been conducted in the edge-on impact test configuration on ultra-high strength concrete in order to induce strong fracturing at the sample rear face in form of spall and tensile cracks. The results provide a unique insight into interactions between microstructural pore network and the dynamic cracking processes such as crack closure and bridging.

\section{Materials and Methods}

\subsection{Experimental setup and X-ray diagnostics}

Experiments were performed exploiting the single-bunch imaging capabilities on ID19 beamline [12, 13] and a single stage gas gun installation available through the user experiment program shown in Figure 2 [14, 15]. The gas gun consists of a breech, $25 \mathrm{~mm}$ bore and a sample environment chamber positioned perpendicular to the X-ray beam with the ability to accelerate the sabot and the flyer up to $900 \mathrm{~m} / \mathrm{s}$ impact speed [16]. The sample chamber is equipped with Mylar windows having a sufficient X-ray transmission as well as stiffness to maintain the vacuum within the sample chamber during the impact. A pair of fast response laser light gates are located at the exit of the barrel which record the impact speed and are used to initiate the triggering of the imaging diagnostics. The trigger sequence consists of a precisely interlaced series of delay generators and coincidence logic gates which ensures the systematic image sequence recording synchronised to the synchrotron bunch clock.

During the experiments, the ESRF operates with 16 bunch filling mode providing a continuous train of bunch electrons with $176 \mathrm{~ns}$ time spacing and pulse width of about $100 \mathrm{ps}$. The X-ray pulses of equivalent time spacing are produced by two undulators set to a minimum gap. The gas gun installation is located in the beamline experimental hutch approximately $145 \mathrm{~m}$ downstream from the source having the detector placed at about $7 \mathrm{~m}$ downstream from the sample. This ensures that the recorded images are within the propagation-based phase contrast regime. The X-ray beam is cropped on the sample using two sets of beamline slits. The transmitted beam is encoded using an indirect ultra-high speed detector set-up consisting of a Shimadzu-HPV X2 ultra-high speed camera and 1:1 Nikon $50 \mathrm{~mm}$ lens arrangement coupled to a $250 \mu \mathrm{m}$ LYSO:Ce fast-decay scintillator (about $40 \mathrm{~ns}$ ). The camera sensor has 400 x 250 effective pixel count with a nominal pixel size of $32 \mu \mathrm{m}$ and on-board memory able to store 128 full frames in a single burst imaging mode. Given the polychromatic beam spectrum with a peak energy of around $30-40 \mathrm{keV}$, the propagation based interference between transmitted X-rays results in an increased contrast of material edges while preserving the material geometrical representation by keeping the fringe effect well within the subpixel 


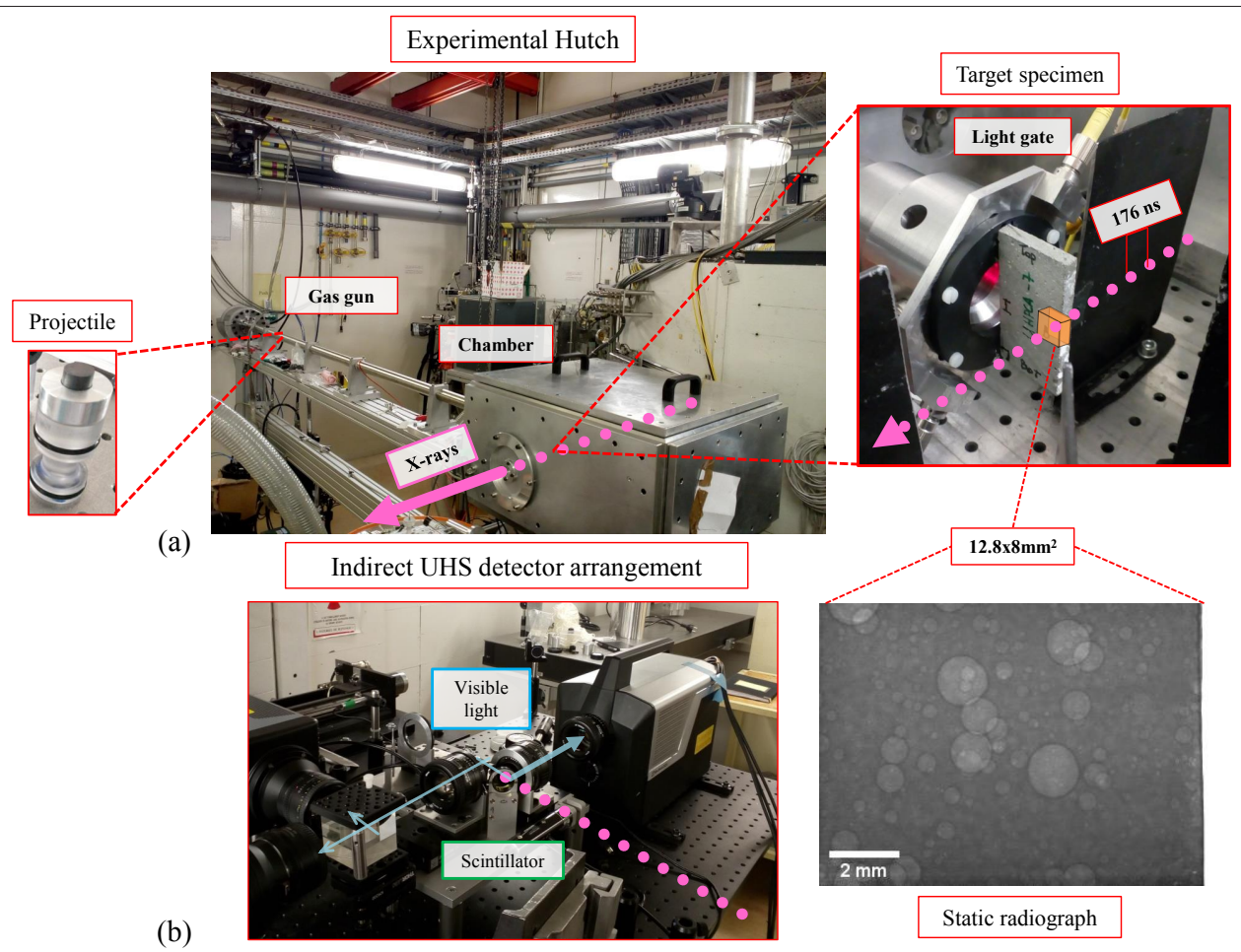

Figure 1: Experimental gas gun setup at ID19: a) Experimental beamline hutch; b) Optical arrangement of the ultra-high speed indirect detector system

range. As a result of the entire optical arrangement, a very large field of view of $12.8 \times 8 \mathrm{~mm}^{2}$ is captured within each radiograph providing the ability to study various transient dynamic phenomena in both solids and liquids within a representative volume scale (e.g. [17.-19]). For the experiments presented herein, the detector acquisition was set to $1060 \mathrm{~ns}$ interframe time with an integration time of $350 \mathrm{~ns}$. This allowed continuous integration over two X-ray bunches for a single radiograph (SNR around 12), which was considered as a good compromise between the amplitude of detected signal and the temporal resolution necessary for sampling the fast fracturing phenomena and following the kinetics of spall fracture formation through the coalescence of cracks.

\subsection{Tested material}

Tested samples consist of an ultra-high performance concrete (UHPC) whose mechanical and microstructural properties differ from a conventional concrete mixture. In contrast to conventional concrete, aside from cement, sand and water, the mixture contains finely crushed quartz grains [9, 23]. The optimised granulometric mixture (maximal grain size of $600 \mu \mathrm{m}$ ) combined with a low ratio of water to cement allows for a relatively homogenous and dense cementitious matrix to be obtained with high mechanical resistance proprieties (Young's modulus around $55 \mathrm{GPa}$ ). The water to cement ratio is 0.21 , after 28-days of curing at $20 \mathrm{C}^{\circ}$ with $100 \%$ relative humidity. This cementitious mix achieves after curing a 
compressive strength exceeding $150 \mathrm{MPa}$ (according to standard NF P 18-470). The tensile strength in bending of this material is about $19 \mathrm{MPa}$ while the dynamic tensile strength is around $35 \mathrm{MPa}$ for strain rates between 100-200 s $\mathrm{s}^{-1}$ [20, 22]. Two prismatic samples $(\mathrm{H}=60 \mathrm{~mm}, \mathrm{~L}=30$ and $\mathrm{t}=6 \mathrm{~mm})$ were tested with two different impact speeds, namely $80 \mathrm{~m} / \mathrm{s}$ and $190 \mathrm{~m} / \mathrm{s}$ within the Edge-On-Impact configuration [24-26]. In both cases, curved cap steel projectile ( $\mathrm{L}=15 \mathrm{~mm}, \mathrm{D}=10 \mathrm{~mm}, \mathrm{R}=100 \mathrm{~mm}$, type 15CDV6) within a dog-bone shaped polycarbonate sabot (total mass $33.8 \mathrm{~g}$ ) were used as a flyer plate to generate incident stress wave in the sample. The relatively short stress wave leads to pronounced compaction and fragmentation at the impact face and dense tensile fracturing at the back face due to scabbing and spall formation. In order to inspect the microstructural porosity distribution before the impact, the samples were scanned with a conventional laboratory X-ray source prior to the experiments. The X-ray source operated at $150 \mathrm{kV}$ and $200 \mu \mathrm{A}$ acquiring a total of 5760 projections during four full-turns in helical scanning mode. At each angle, four radiographs acquired at two frames per second were averaged together to reduce noise. In this way, full sample dimensions were imaged with a resulting voxel size of $30 \mu \mathrm{m} / \mathrm{vx}$. The software XAct was used to reconstruct 3D volume from the captured radiographs using a filtered back projection algorithm and Tukey filter kernel. The porosity map was segmented from reconstructed volumes using the Otsu threshold method. Then a series of morphological operations (i.e. watershed, equivalent sphere and ellipse analysis) were performed using the label toolbox in the open source Python software spam [27] as to obtain the porosity distribution shown in Figure 2. The results showed that less than $20 \%$ of porosity consisted of pores with a diameter larger than $500 \mu \mathrm{m}$. The resulting volume analysis indicated an overall porosity of the samples being around $2.5 \%$ of the total volume.

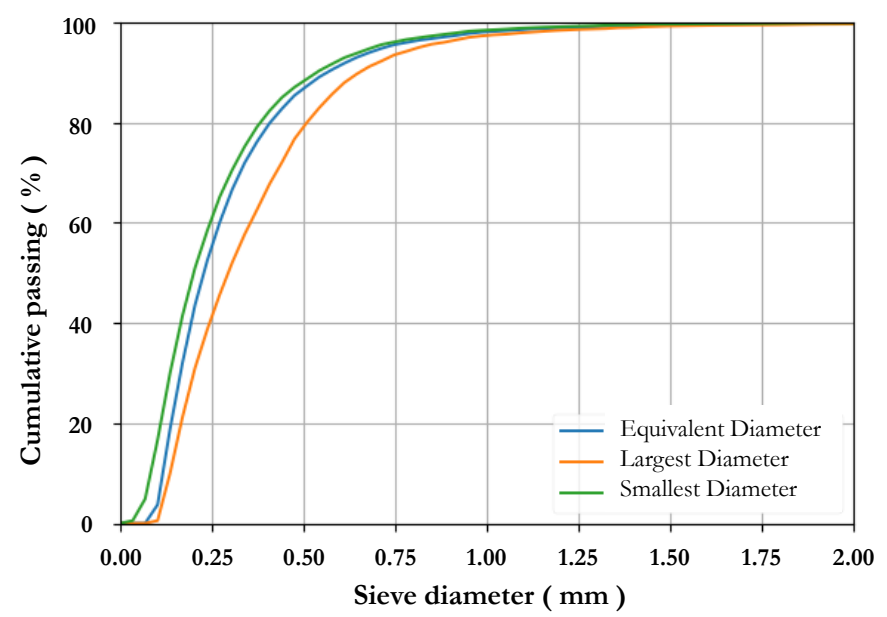

Figure 2: Example of a porosity distribution in the UHPC sample obtained from X-ray computed tomography analysis before impact experiments. (The smallest and largest diameter are obtained with equivalent ellipse analysis [28])

\section{Results}

The recorded radiography sequences of each experiment were first corrected by removing outliers introduced by any potential scattering from the surroundings. Then the flat field 
and dark field corrections were performed using the flat field image sequence captured straight after the test shot. The time sequences of captured UHS-XPCI radiographs on two tested samples are shown in Figures 3 and 4 . For the case of the specimen impacted with a lower impact speed of $90 \mathrm{~m} / \mathrm{s}$, several tensile cracks are observed to initiate at the sample free-end (horizontal cracks in Figure 3). These cracks seem to propagate following a path of higher porosity content and finally forming a fragment that surrounds the volume of a lower porosity content. Similarly, in the case of the sample impacted with $190 \mathrm{~m} / \mathrm{s}$ impact speed, a tensile crack initiates at the sample free-end and tends to follow the path towards the higher porosity content. However, a vertical spall fracture seems to form at the approximately same time due to a sharper loading pulse. The spall crack seems to be perfectly vertical without any pronounced influence of the local surrounding porosity content on its path. Similar observations were made in uniaxial Hopkinson bar spalling experiments with post-mortem analysis [21]. Furthermore, an interesting observation is that during the subsequent fracture evolution, the spall crack completely closes due to secondary stress wave and gets completely bridged by the continuously propagating horizontal tensile fracture as such as within a continuous medium.

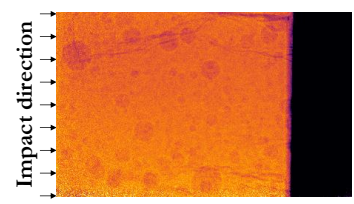

(a) $t=T_{0}+25.44 \mu \mathrm{s}$

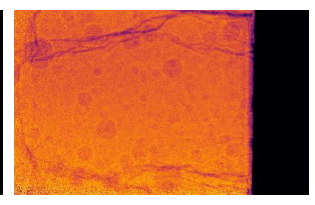

(b) $t=T_{0}+36.04 \mu \mathrm{s}$

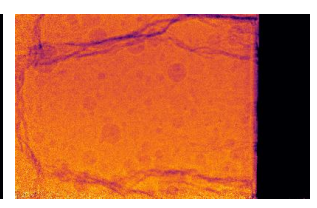

(c) $t=T_{0}+44.52 \mu \mathrm{s}$

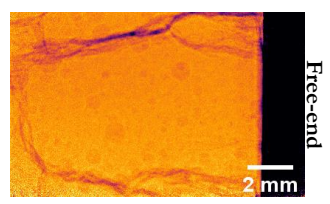

(d) $t=T_{0}+65.72 \mu \mathrm{s}$

Figure 3: XPC radiographs captured during in-situ edge-on-impact test on an ultra-high strength concrete sample impacted with $80 \mathrm{~m} / \mathrm{s}$, showing the initiation of tensile cracks at free-end and progressive coalescence to form spall fragments. (Impact direction is from the right hand side.)

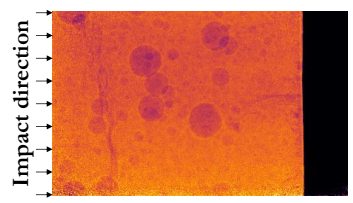

(a) $t=T_{0}+21.2 \mu \mathrm{s}$

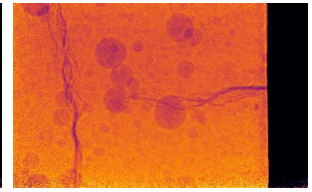

(b) $t=T_{0}+31.8 \mu \mathrm{s}$

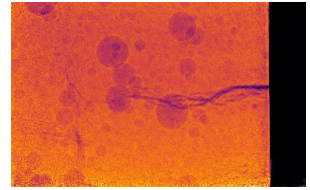

(c) $t=T_{0}+42.4 \mu \mathrm{s}$

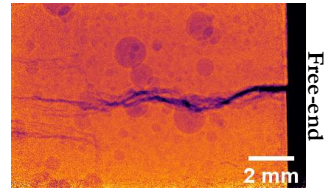

(d) $t=T_{0}+63.6 \mu \mathrm{s}$

Figure 4: XPC radiographs captured during in-situ edge-on-impact test on an ultra-high strength concrete sample impacted with $190 \mathrm{~m} / \mathrm{s}$, showing the opening, closing and bridging of the spall fracture at the sample rear face due to the secondary stress wave. (Impact direction is from the right hand side.)

In order to verify the presence of the secondary stress wave, analysis of the rear face particle velocity profile deems necessary. However, due to the difficulties of incorporating point-wise probe measurements based on laser interferometry, image analysis was used to obtain the space-time evolution of the rear face horizontal velocity component at the sample free-end. The squared variance of transmittance is used to isolate the sample free edge in each radiography. Then, a signal processing approach was adopted assuming that the edge does not substantially deform within $100 \mu \mathrm{m}$ from the sample free-end. The horizontally sampled variance signal is then followed in time and at each vertical pixel position by maximising the normalised cross-correlation product between subsequent frames. A Gaussian fit 
is performed on the distribution of spatial lags where the mean is directly related to the differential horizontal displacement between two radiographies on a sub-pixel level. Finally, the Savitzky Golay polynomial fit of order two over five time points was used to derive the temporal distribution of cumulative displacement to obtain the horizontal velocity field depicted in Figure 5. The space time velocity fields clearly demonstrate that on the same time scale, the sample impacted with higher impact speed shows the arrival of the second stress wave at about $50 \mu \mathrm{s}$. Finally, the average velocity on the entire free surface is shown in Figure $5 \mathrm{c}$ This information is often used for inverse calibration of phenomenological numerical models.

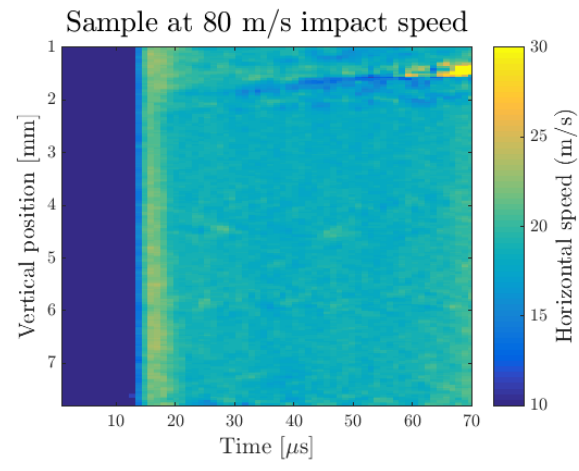

(a)

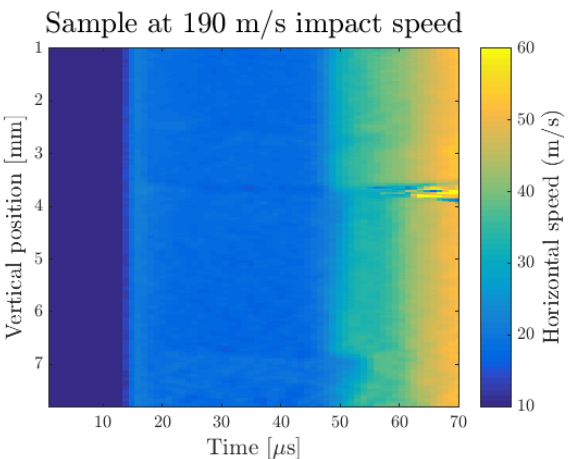

(b)

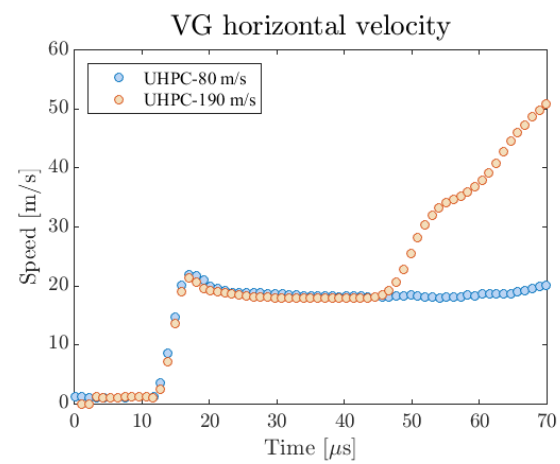

(c)

Figure 5: Space-time evolution of the rear face velocity profile for two tested samples.

\section{Conclusions}

In this work, the dynamic fracturing of an ultra-high strength cementitious material is probed with in-situ ultra-high speed synchrotron radiation X-ray phase-contrast imaging to visualise the dynamic through-volume fracturing. Gas gun experiments were conducted on two characteristics samples with two different impact speeds and results revealed the phenomena of a formation of a spall fracture in parallel to dense tensile fracturing from the sample freeend. For the case of impact at higher speed, the spall fracture closure occurred allowing to be bridged by a perpendicular propagating tensile crack. The direct observation of these 
processes is pivotal in better understanding the leading physical mechanisms involved in dynamic fragmentation and improving the performance of the representative numerical models.

\section{References}

[1] F. Hild, C. Denoual, P. Forquin, X. Brajer, Comput. Struct. 81, 1241-1253 (2003)

[2] F. Hild, Mech. Mater. 80, 340-350 (2015)

[3] A. Rosakis, O. Samudrala, D. Coker, Science 284, 1337-1340 (1999)

[4] K. Ravi-Chandar, Dynamic Fracture, (Elsevier Science, 2004)

[5] P. Forquin, F. Hild, Adv. Appl. Mech. 44, 1-72 (2010)

[6] L. Pereira, J. Weerheijm, L. Sluys, Eng. Fract. Mech. 182, 689-707 (2017)

[7] J. Bleyer, C. Roux-Langlois, J.-F. Molinari, Int. J. Fract. 204, 79-100 (2017)

[8] L. Rozen-Levy, J. M. Kolinski, G. Cohen, J. Fineberg, Phys. Rev. Lett. 125, 175501 (2020)

[9] P. Forquin, F. Hild, J. Eng. Mech. 4, 302-315 (2008)

[10] M. P. Olbinado, X. Just, J.-L. Gelet, P. Lhuissier, M. Scheel, P. Vagovič, T. Sato, R. Graceffa, J. Schulz, A. Mancuso, J. Morse, A. Rack, Opt. Express 25, 13857-13871 (2017)

[11] E. Escauriza, M. Olbinado, M. Rutherford, D. Chapman, J. Jonsson, A. Rack, D. Eakins, Appl. Opt. 57, 5004-5010 (2018)

[12] A. Rack, M. Scheel, L. Hardy, C. Curfs, A. Bonnin, H. Reichert, J. Synchrotron Rad. 21, 815-818 (2014)

[13] M. Olbinado, A. Rack, Proc. SPIE 11051, 110510F (2019)

[14] M. Rutherford, D. Chapman, J. Derrick, J. Patten, P. Bland, A. Rack, G. Collins, D. Eakins, Sci. Rep. 7, 1-9 (2017)

[15] E. Escauriza, J. Duarte, D. Chapman, M. Rutherford, L. Farbaniec, J. Jonsson, L. Smith, M. Olbinado, J. Skidmore, P. Foster, A. Rack, D. Eakins, Sci. Rep. 10, 8455 (2020)

[16] D. Eakins, D. Chapman, Rev. Sci. Instrum. 85, 123708 (2014)

[17] N. Bourne, W. Mirihanage, M. Olbinado, A. Rack, Sci. Rep. 10, 10366 (2020)

[18] M. Olbinado, V. Cantelli, O. Mathon, S. Paskarelli, ..., A. Rack, J. Phys. D: Appl. Phys. 51, 055601 (2018)

[19] S. Bornschlegel, C. Conrad, A. Durst, R. Welss, M. Wensing, M. Olbinado, L. Helfen, T. Baumbach, Int. J. Engine Res. 2, 592-605 (2019)

[20] M. Blasone, D. Saletti, J.Baroth, P.Forquin, E. Bonnet, A. Delaplace Int. J. Impact Eng. 152, 103838 (2021)

[21] M. Blasone, D. Saletti, E. Andò, J. Baroth, P. Forquin, EPJ Web. Conf. 183, 03024 (2018)

[22] M. Blasone, Experimental testing and numerical modelling of the tensile and compression damage in Ultra-High Performance Concrete under impact loading, (Universite Grenoble Alpes, 2021)

[23] P. Forquin, B. Lukić, Dynamic Behaviour of Materials, Volume 1, (Springer International Publishing, 2016) 229-237

[24] E. Strassburger, H. Snef, H. Rothenhausler, J. Phys. IV 4, C8 (1994)

[25] P. Forquin, L. Tran, L. Louvigné, F. Hild, Int. J. Impact. Eng. 28, 1061-1076 (2003)

[26] P. Forquin, B. Lukić, Y. Duplan, D. Saletti, D. Eakins, A. Rack, Proc. SPIE 11051, 110510Q (2019)

[27] O. Stamati, E. Andò, E. Roubin, R. Cailletaud, ..., G. Birmpilis, J. Open Source Softw. 5, $2286(2020)$

[28] S. Ikeda, T. Nakano, Y. Nakashima, Y. Miner. Mag 64, 945-959 (2000) 